\title{
A Release Device for Parachute and Robot
}

\author{
Xuanyang Shi ${ }^{1, \text { a }}$, Junyao Gao*1, b ,Jingchao Zhao ${ }^{1, \mathrm{c}} \mathrm{Yi} \mathrm{Liu}^{1}$, Fangzhou \\ Zhao ${ }^{1}$,Haoxiang Cao ${ }^{1}$ and Chuzhao Liu ${ }^{1}$ \\ ${ }^{1}$ School of Beijing Institute of Technology, Beijing 100081, China; \\ a313585956@qq.com, bgaojunyao10@163.com, czhaojingchao88@126.com \\ Corresponding Author: Junyao Gao
}

Keywords: Release device, Acceleration

\begin{abstract}
Parachute release device can be used in airdropping rescue robots when emergencies like earthquake and nuclear leak occur. The problem of inaccuracy exists in traditional purely mechanical design and time delay mechanism. In order to solve this problem, it is urgent to develop a suitable release device.

In this paper, we designed a new type of release device based on acceleration sensor. Analysis the acceleration of the airdrop procedure, establish an acceleration model of the entire process, make the judgment of separate. We designed an integral mechanical structure which remains nothing on robot after separated. At last, experiment proved that the release device we designed can separate correctly.
\end{abstract}

\section{Introduction}

Reconnaissance and surveillance are the most important activities for both military and civilian organizations [1]. When the earthquake, chemical gas leak, radiation-contaminated happened, the aerial dispersal miniature reconnaissance robots can be deployed in sensitive region by air-delivering and they are easier to intrude spatially confined spot and evade detection than larger robots[2 5]. But, sometimes the robot can't separate from the parachute in time, or never separate, leading that the robot can't work and delay the rescue activity. Therefore, a reliable release device is needed to separate the robot from the descent system in rescue activities [6 7].

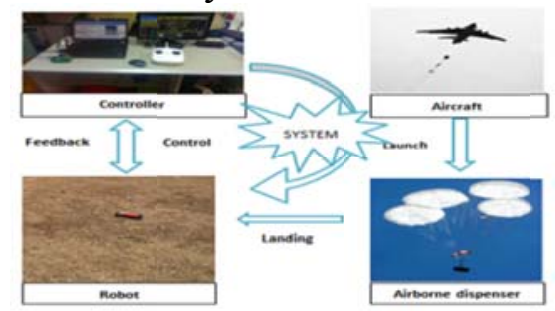

Fig.1 The aerial dispersal miniature reconnaissance robot system

The overall dispersing process of robot is shown in Fig.1.The robot is mounted in the Air jettisoning system which is mounted in the plane. After launched, the robot, release device and parachute will leave the cabin rapidly under the effect of catapult and gravity. Then, the release device works to separate robot and parachute after landing, and the robot comes into operation under the control of controller, moving, obtaining location information, taking photos, et

Parachute release device is used for cargo airdrop widely. There have been some studies on this aspect at home and abroad. The Atlas PR-A2 Parachute Release is a mechanism that separates the cargo from its parachute by sensing a reduction in the suspended weight upon ground impact accurately, the weight is $1.5 \mathrm{~kg}[8-9]$. The FXC release device separate the cargo when subjected to impact[10]。 The two devices are mechanical design, only taking the landing impact as single separation criteria. Consequently, it is highly possible to make false positives caused error 
separation in service. Besides, after mechanical separation, part of it is left on the cargo, and it will affect the robot to perform tasks. Existing mechanical products can't meet the demands. So we designed a new release device which can overcome these difficulties. The new device has the following functions: Firstly, when the release device reaches the designated airspace, it will be charged automatically by a mechanism. In this way, it can avoid misjudgment and erroneous separation caused by service operation or flying process. Secondly, it can work at different height and mass. Thirdly, The mechanism of the release device is reliable and can meet the requirements of the task in a complex environment. Finally, It can be installed quickly and it's convenient to use it without complex external auxiliary equipment needed.

We designed and manufactured a general and portable release device with high suitability, high accuracy, high stability, and simple operation suitable for after-calamity regions with high risks, such as crucial spots, hostage and survivor rescue, illicit drug raids, and responses to nuclear or chemical or toxic waste contamination [11-12].

\section{Manufacturing of the release device}

\subsection{Mechanism of release device}

Fig. 2 is the mechanism of release device. There is a movable slipper in the chute of Chassis, the slipper slide internal and external under the actuator. Fig. 3 shows the slider's outermost limit position. In this time, the cargo is jammed by slider and as together. Fig. 4 shows the slider's innermost limit position. At this point, all the sliders slide into the innermost limit position and the cargo separates from the release device.
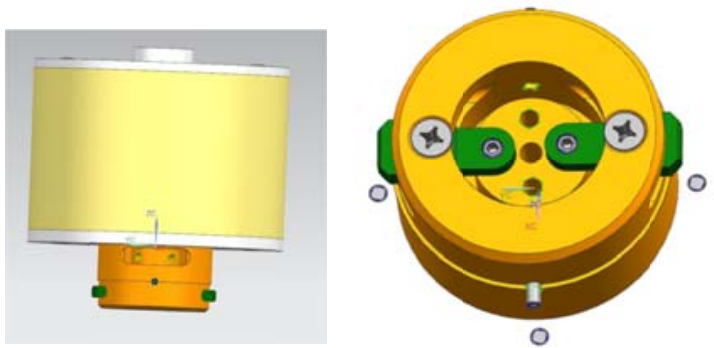

Fig. 3 outermost limit position

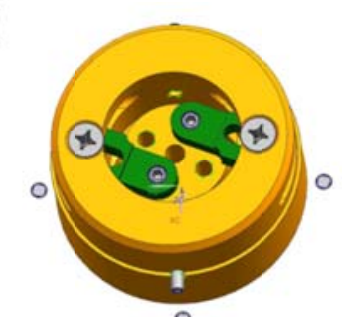

Fig.4 innermost limit position

Fig.2 Overall structure

\subsection{Release device strength safety}

In the release device, the strength of the movable slider and the chassis determine the security of the whole.

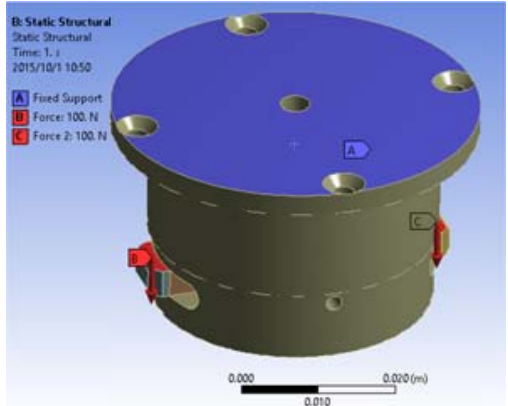

Fig. 5 static structure

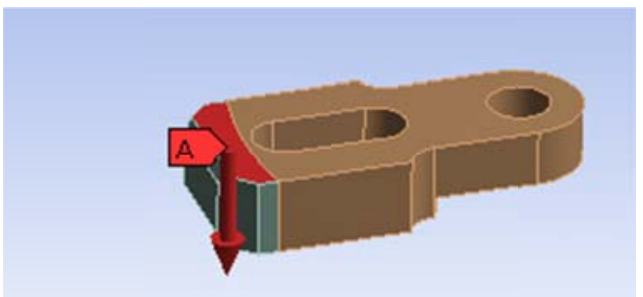

Fig.6 slider structure

In Fig.5, label A represents the top surface of the Chassis of Full Constraint Solving. Label B and $\mathrm{C}$ represent infliction of $10 \mathrm{~kg}$ force on the top surface of the two sliders. The release device carry about 2KG cargo as shown in Fig.6.The interface of the movable slider and Chassis is a movable contact surfaces, and the slider can slide and separate from the interface. So the boundary conditions of the working parts of the release device are described in the finite element model.

The stress contour of the release device is illustrated in Fig.7. The figure shows that the maximum Von-Mises stress is $40.4 \mathrm{MPa}$, and the yield stress of the selected material is $235 \mathrm{MPa}$, namely the safety performance of the whole structure is adequate. 


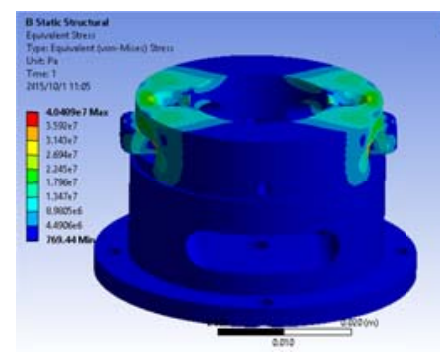

Fig.7 stress contour figures

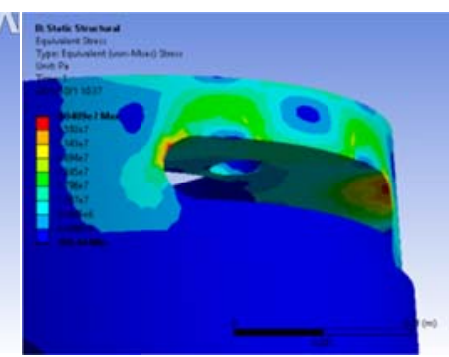

Fig. 8 the chute

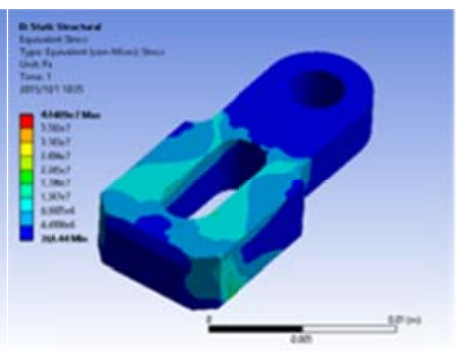

Fig. 9 slider's stress distribution

Fig. 8 is the chassis of the release device, which shows that the stress is mainly distributed in the contact of the slide rand, and the maximum stress is $40.4 \mathrm{MPa}$. These diagrams show that the chassis is fully capable for meeting the requirements.

Fig.9 show the slider's stress distribution and the maximum stress is $40.7 \mathrm{MPa}$.It meets the requirements too. Fig 10 is the manufactured mechanical structure which shows the outermost limit position and the innermost limit position.

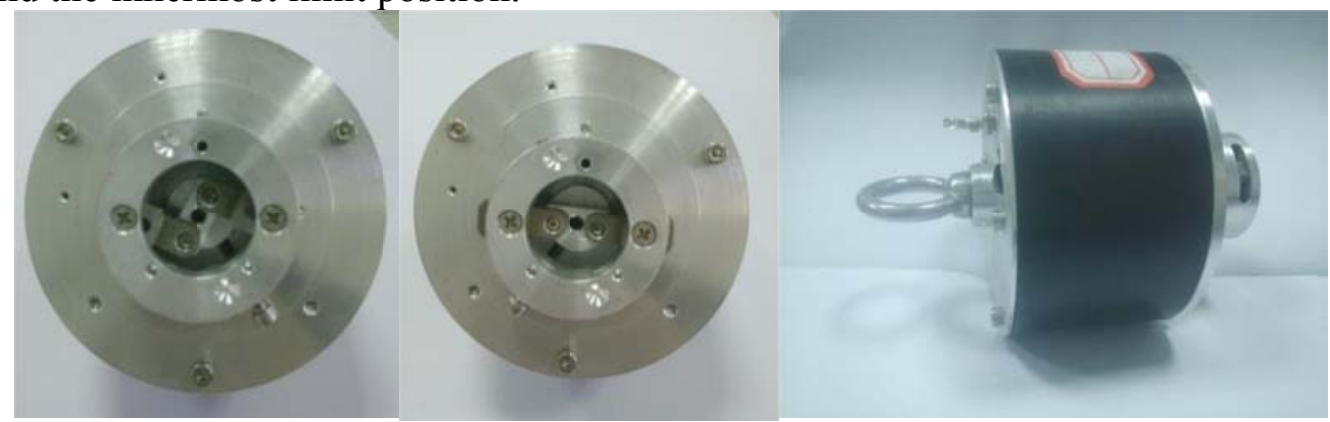

Fig.10 parachute release device

\section{Data acquisition and analysis and design of released method}

\subsection{Data acquisition unit(DAQ) and robot launcher.}

In the flying and landing process, the parachute, release device and robot(PDR) are connected together as a whole. When the robot landed, the release device and the parachute would separate from it. In order to study the motion characteristics of the robot landing, a data acquisition system is designed to collect the acceleration characteristics of the whole process.

The DAQ system consists of a parachute, DAQ unit, robot model, and the launcher. In the DAQ-experiment, the robot model is used to replace the robot in different environment.

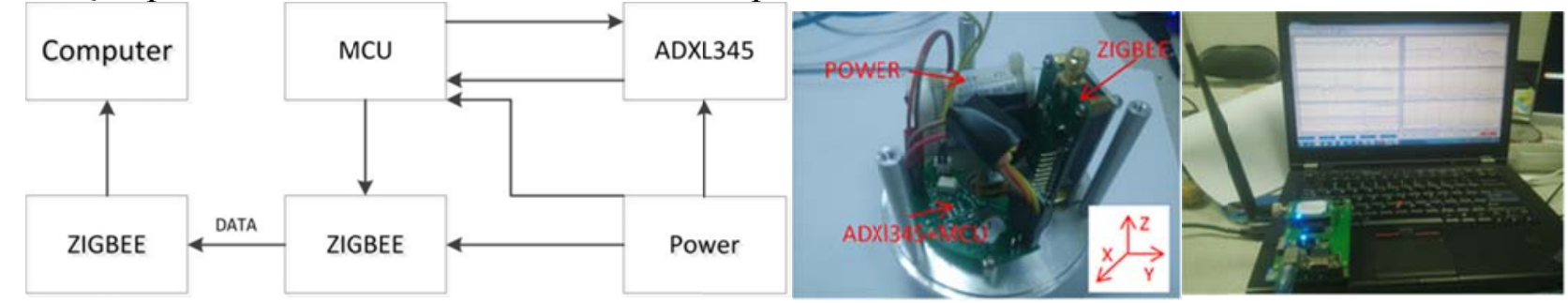

Fig.11 DAQ system

This Data acquisition unit can collect the data of the robot and release device during the descent and landing. It is consisted of MCU, ZIGBEE, battery and ADXL345 digital accelerometer. Firstly, the accelerometer collects the acceleration of the PDR which is consisted of Parachute, DAQ unit and robot model, and transfers these data to the MCU by serials. Secondly, the MCU transfers these data to a ZIGBEE. Finally, the computer receives data by another ZIGBEE, and the data can be processed and analyzed in the computer.

We used the DAQ system to collect the accelerated speed in the process of airdropping. Fig.12 shows the acceleration collection experiment. As illustrated in Fig 12, the experiment was carried out on a building of 10-meters in height. In this height, all steps of a real airdrop can be simulated in the experiment, including launching, opening parachute and landing processes. 

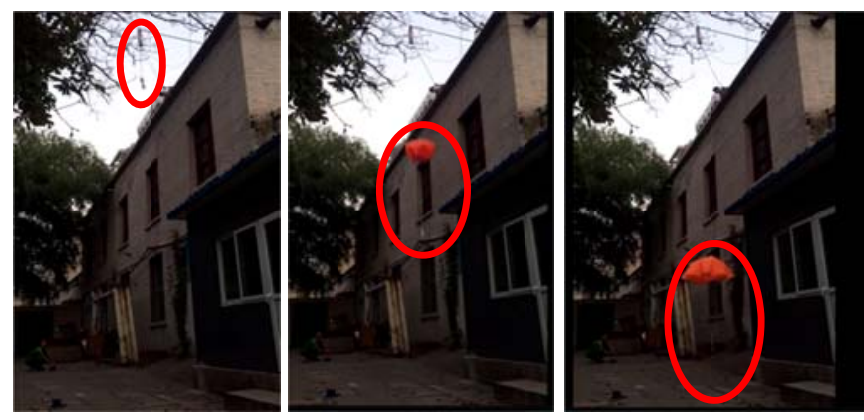

Fig.12 DAQ-experiment

\subsection{Characters of acceleration.}

In order to get the acceleration data curve for obtaining the criteria to determine whether the robot has separated from the PDR, the DAQ system launches the PDR to a position high enough and collects the acceleration data at the same time, shown in Fig.13. The position is about 11 meters in height, which is high enough for the parachute opening and descent.
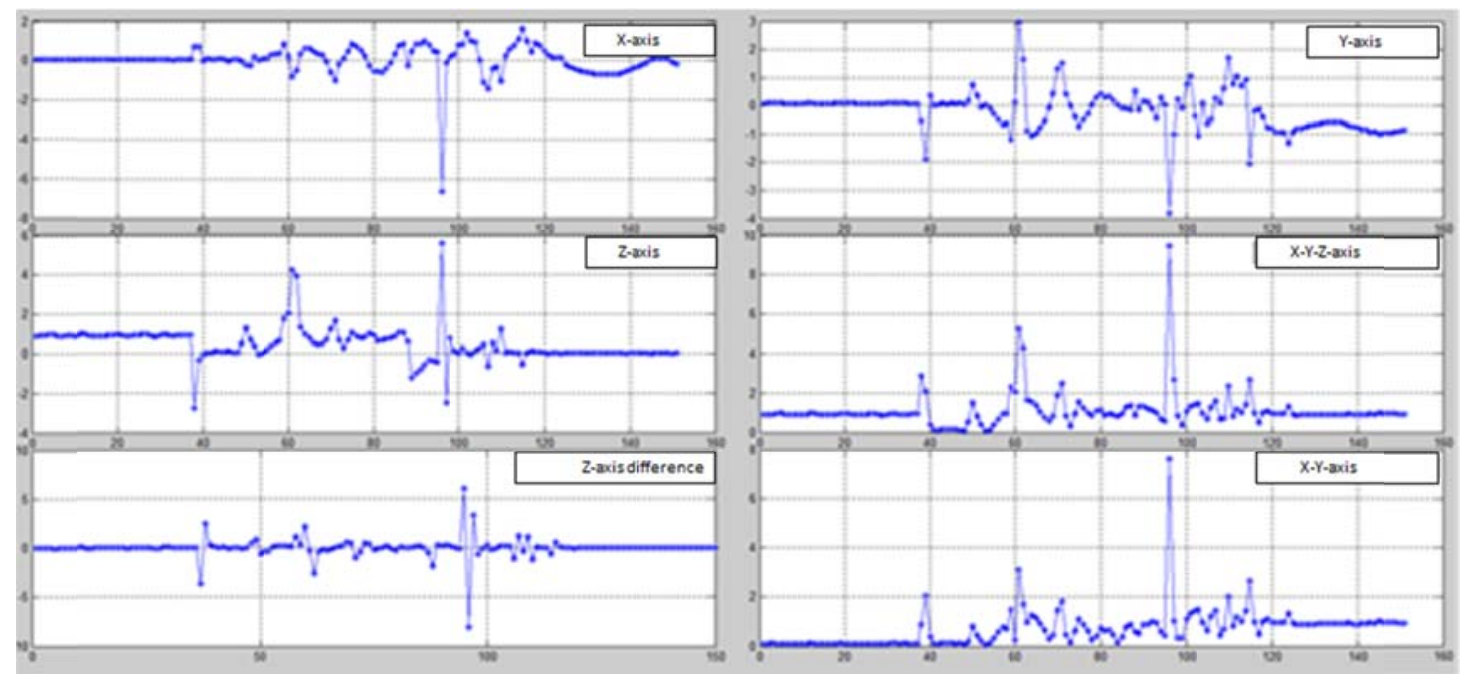

Fig. 13 The acceleration during the PDR descent and landing

The acceleration curve shows that the $\mathrm{z}$-axis acceleration value is about $1 \mathrm{~g}$. When the DAQ system is hanging in the air, and the acceleration peak appears four times in the descent step.

For the Z-axis, the first peak is negative because of the pushing by the spring to the PDR. When the robot connected with the DAQ unit has been pushed out of the launcher, the parachute was still on it, and needed to be pulled out of it, which causing the second peak. After the parachute got out of the launcher, it would open quickly and the third peak appeared in the acceleration curve, which is the biggest one among the three peaks. Obviously, the PQR will be pulled up by the opening parachute and descent-pulled for several times until it is close to uniform descent (the z-axis acceleration value is about $1 \mathrm{~g}$ ). When the robot impacts the ground, the acceleration will rise immediately and be fluctuant for a while until it is close to static.

For the $\mathrm{x}$-axis and $\mathrm{y}$-axis acceleration, the value is about $0 \mathrm{~g}$ while the $\mathrm{z}$-axis is about $1 \mathrm{~g}$. The acceleration values are fluctuant and have no obvious characteristics during PQR's descent and landing, and will be close to a stable value when PQR land on the ground. Then the $x$-y-axis resultant acceleration becomes about $1 \mathrm{~g}$ because the DAQ unit and robot model (QR) attitude are horizontal

Fig13 also shows the $\mathrm{x}-\mathrm{y}-\mathrm{z}$-axis resultant acceleration, whose characteristics are similar to $\mathrm{z}$-axis, but the acceleration is resultant and not reveal the acceleration direction.

\subsection{Criteria for releasing.}

According to the acceleration data of every stage, we can make a table. 
Table 1 acceleration data of every stage

\begin{tabular}{cclll}
\hline state & $\mathrm{X}(\mathrm{g})$ & $\mathrm{Y}(\mathrm{g})$ & $\mathrm{Z}(\mathrm{g})$ & $\mathrm{X}-\mathrm{Y}-\mathrm{Z}(\mathrm{g})$ \\
\hline flying & 0 & 0 & 1 & 1 \\
lunching & $0.7-0.9$ & -2 & -2.5 & 2.9 \\
Opening parachute & -1 & 3 & 4.2 & 5.4 \\
falling & $-0.3-0.3$ & $-0.3-0.4$ & $0.8-1.3$ & $0.8-1.4$ \\
landing & -6.5 & -3.9 & 5.8 & 9.5 \\
after landing & 0 & -1 & 0 & 1 \\
\hline
\end{tabular}

In table 1, we can put this process into two types: steady state and mutation status. As is shown in table 1, states in flying, falling and after landing are marked yellow which indicates a steady state. In the same way, launching, opening parachute and landing are marked red which indicates mutation status. Their combination and cross application make us able to determine the state of PDR throughout the process.

During flying, the PDR is mounted vertical in the Air jettisoning system, so the PDR's Z-axis is $1 \mathrm{~g}$, and both the $\mathrm{X}$-axis and $\mathrm{Y}$-axis are $0 \mathrm{~g}$,namely the first criteria are $-0.1<\mathrm{x}<0.1-0.1<\mathrm{y}<0.1,0.9<\mathrm{Z}<1.1$. The first peak generated in the launching state. Affected by spring and gravity, the $\mathrm{Z}$-axis peak is negative. Meanwhile, the $\mathrm{X}$-axis and $\mathrm{Y}$-axis data produced by the collision between the robot and the wall or disturbances can't be used as the non-characteristic data, so the second criteria is $\mathrm{z}<-2.4$. When the parachute is opening, we get a positive peak because the robot is pulled up by an upward force. Under the interference from external conditions, such as wind, each of the three axis of acceleration is uncertain, but the resultant acceleration can be determined almost. Therefore, the third criteria is $\mathrm{X}-\mathrm{Y}-\mathrm{Z}>5.3$. The falling state is as following. When the PDR reaches uniform state, the acceleration would be fluctuating between $0.8 \mathrm{~g}$ and $1.4 \mathrm{~g}$. In order to distinguish the state after landing, the Z-axis is used as secondary criteria, so the forth criteria is $0.8<\mathrm{Z}<1.3,0.8<\mathrm{X}-\mathrm{Y}-\mathrm{Z}<1.4$. When the PDR touch down, PDR will get the third peak because of the strong impact. But, just like the state of opening parachute, each of the three axis of acceleration is uncertain which is caused by the uncertain contact face between robot and ground. Therefore, the fifth criteria is $Z>9.3$. After landing, the robot will fall down. But if the angle of floor is not greater than 45 degree, the $\mathrm{Z}$-axis will not be greater than $0.7 \mathrm{~g}$ and the $\mathrm{X}-\mathrm{Y}-\mathrm{Z}$-axis will be $1 \mathrm{~g}$. Hence, the sixth criteria is $\mathrm{Z}<0.7,0.9<\mathrm{X}-\mathrm{Y}-\mathrm{Z}<1.1$.

\subsection{Releasing algorithm design.}

Based on the above criteria, it can be designed in the following flow chart. In Fig.14, we use the criteria 2nd to 6th mentioned above to establish the entire dumping procedure. Identify the threshold of acceleration to determine each process. In the recognition of the falling state, acceleration values satisfy the condition once it can't represent a steady falling, so we have to recognize it twice. When the acceleration satisfies the pre-set process, it means that the PDR has landed, and then the release device would drive the motor to separate robot and parachute automatically, and two seconds later, stop it automatically. 


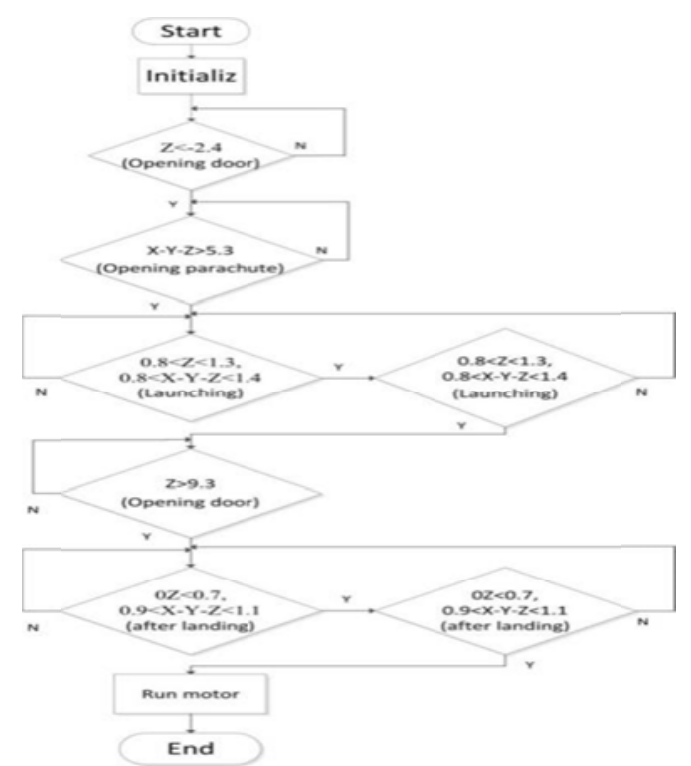

Fig.14 Flowchart

\section{Landing experiment of release device}

\subsection{Experimental Process}

After ground joint debugging, in order to verify the design of release device, we use the PDR system to replace the DAQ system and take the experiment again, just like the acceleration collect experiment.

As show in Fig.15 and Fig.16.The first step, connect the parachute, release device and robot. The second step, put the PDR into launcher and lock the launcher door. The third step, hang the airdrop system in 10 meters building. The last step, open the launcher by manual control.

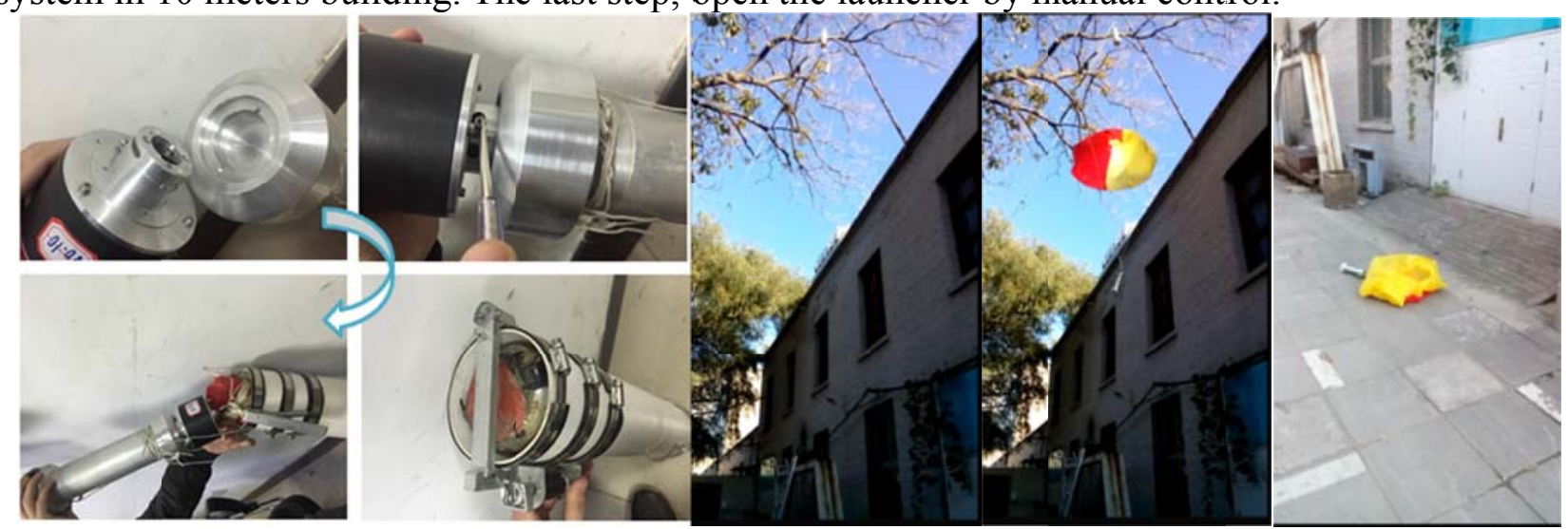

Fig.15 The installation of ejector

\subsection{Experimental Results}

Fig.16 The experiment of airdrop

The results of experiments demonstrated that: the robot, release device and robot were successfully launched. The parachute can be fully deployed in the falling process. The robot and release device can be connected reliably in falling process and separated from each other after landing .In the whole experiment, the robot and release device didn't separate too early or never. So the release device designed in this paper can meet the requirements of the task.

\section{Conclusion}

Aiming at overcoming the shortcomings of the existing parachute release device which may tend to cause misjudgment and leave part of the release device on the robot after separated, we designed a new type of release device basing on acceleration sensor. Set threshold according to the three acceleration parameter. Determine the moving state by analysis the acceleration data. The algorithm 
adapt to the complex task environment very well. At the same time, the release device improves the design method by the two part of combination, which solves the problem of leaving part of the release device on robot. Meanwhile, the release device not only can be used in two-wheel robot, but also in six-wheel robot and so on. It has laid the foundation of the application of the release device.

\section{Acknowledgements}

This work was supported in part by the National Key Technology R\&D Program of China under Grant 2013BAK03B03, the "111 Project" (Grant No. B08043), National Science Foundation for Distinguished Young Scholar (Grant No. 60925014) and National Natural Science Foundation of China 61175077.

Xuanyang Shi, Junyao Gao and Jingchao Zhao are with the Intelligent Robotics Institute, School of Mechatronical Engineering, Beijing Institute of Technology, Nandajie, Zhongguancun, Haidian, Beijin,100081,China(corresponding author to provide email: gaojunyao10@163.com).

\section{References}

[1] D. F. Hougen, S. Benjaafar. "A Miniature Robotic System for Reconnaissance and Surveillance ", Proc. of IEEE Int' 1 Conf. on Robotics and Automation, San Francisco, U.S.A., 2000: 501-507.

[2] Nadir R. Parachute release device for unmanned aerial vehicle (UAV): US, US 8191831 B2[P]. 2012.

[3] Yuan X, Chen D, Tian X, et al. Three-axis digital accelerometer ADXL345 and its application in SINS design[J]. Electronic Design Engineering, 2010.

[4] Nadir R. PARACHUTE RELEASE DEVICE AND METHODS: WO, EP1945508[P]. 2008.

[5] Xiao Q, Chen Q. Ultra-low-power Angle Measurement Instrument Designed Based ADXL345[J]. Computer Knowledge \& Technology, 2014.

[6] Bejtlich Iii C L. Pressure dependent parachute release device for air/water rockets: US, US 5785278 A[P]. 1998.

[7] Nadir R. Parachute release device for unmanned aerial vehicle (UAV): US, US 8191831 B2[P]. 2012.

[8] Nadir R. PARACHUTE RELEASE DEVICE AND METHODS: WO, EP1945508[P]. 2008.

[9] S. A. Stoeter, P. E. Rybski, K. N. Stubbs, et al, “A robot team for surveillance tasks: design and architecture", Robotics and Autonomous Systems, Elsevier, 2002,40(2), pp.173-183

[10] THURNER GUENTHER D R, Helmut H. Ejectable body with parachute - esp. submunition with reliable parachute release device: , DE 4023628 A1[P]. 1992.

[11] S. A. Stoeter, P. E. Rybski, K. N. Stubbs, et al, "A robot team for surveillance tasks: design and architecture", Robotics and Autonomous Systems, Elsevier, 2002,40(2), pp.173-183

[12] S. J. Hsieh and P. Y. Hsieh, "Web - based modules for programmable logic controller education," Computer Applications in Engineering Education, vol. 13, pp. 266-279, 2005. 